Besides these there were a few other small clouds, white, and of unusual character and brightness, but at no time did I detect in them any certain trace of colouring.

December 3I.-At about ro a.m., and for some time after, I noticed a small coloured cloud, above, but a little to the west of, the sun, and at an altitude of about $30^{\circ}$. The lower edge was blue, immediately above this a narrow band of green, the rest of the cloud being faint, almost colourless, but occasionally showing a slight yellowish tinge, and at the upper edge a touch of red.

At sunset there was the most wonderful display. At $4 \mathrm{p} . \mathrm{m}$. in all parts of the sky were bands of light cirrus cloud, flushed with crimson by the setting sun. In the west, higher than the cirrus, and shining through them and in the intervals between, were splendid iridescent clouds, set off against a background of blue. I have never yet seen anything to equal the glory of this sky. It is impossible to describe it, the colours were so varied and their changes so rapid. I confined my attention, therefore, to the two largest and most beautiful clouds, and the following is a record of the colours and their variations, so far as I was able to observe them.

(I) One of these clouds is shown in Fig. I. It was situated a few degrees south of west, about $20^{\circ}$ above the horizon, and was about $15^{\circ}$ in length and $5^{\circ}$ in greatest breadth. During the whole time it seemed stationary, though changing slightly in

Fig.2.
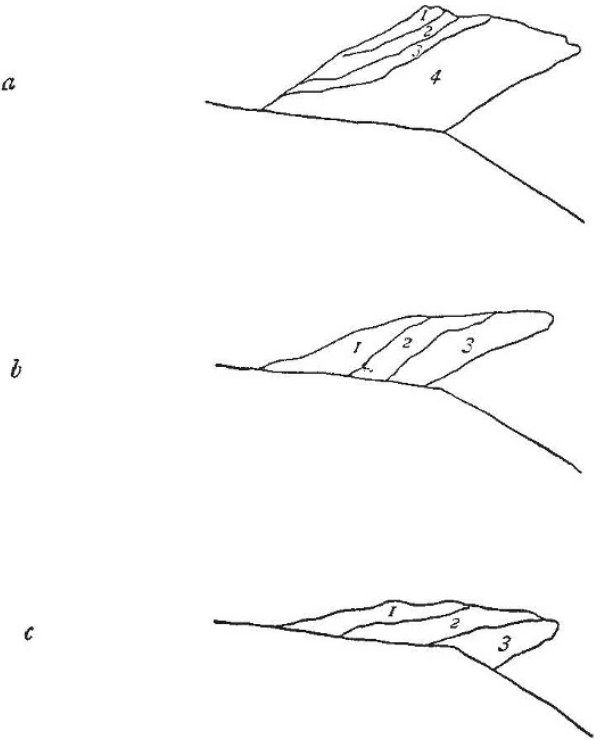

form. It was crossed by beautiful bands of colour, separated by fairly sharp lines. Sometimes, when the stripes of colour were narrow, I have included them in one band, to save time in drawing; the order being then given from left to right.

4.5p.m.-Fig. I, a. The right end of the cloud hazy. (I) Green, (2) yellow, orange, red; (3) blue, green, yellow, red; (4) red, with a tinge of purple ; (5) bright purple; $(6)$ red, green.

4. II. - Fig. I, $b$. Both ends hazy. (1) Pink in the haze, blue ; (2) yellow, green, orange : (3) green; (4) red ; (5) blue ; (6) red, ending in green in the haze.

4. I8.-Fig. I, c. The cloud rather longer and narrower than before. (1) Green ; (2) green; (3) yellow, orange ; (4) green; (5) red; (6) green; (7) red.

4.21. - The orange band 3 beginning to invade the green band 2.

4.22. The red band 5 growing brighter.

4.25. - The red and orange bands, 3 and 5, widening, and becoming the predominant colours of the cloud.

4.27.-Fig. I, $d$. (1) Light haze ; (2) hazy; (3) red; (4) green; (5) bright red; (6) greenish; (7) orange red. The general hue of the cloud was at this time reddish orange, the red and orange bands being much brighter than the rest.

4.30.-Fig. I, e. The outline hazy. (I) Haze; (2) red : (3) reddish orange; (4) red.
4.32.--Fig. I, f. (1) Thin haze; (2) not quite so bright as before, but all of a deep rose-c slour.

4.34. - The rose-coloured part had a slight tinge of purple, which, two minutes later, had become more marked, but rising clouds now stopped further observation.

(2) The other cloud was in a west-south-west direction, about I0 above the horizon, and partly hidden by a bank, which served, however, to show how slightly the cloud altered its position.

4.7 p.m.-Fig. 2, a. (I) Green; (2) orange red; (3) light green ; (4) violet. All these colours very bright.

4. 13.- The colours had changed, so that the general hue of the cloud was blue.

4. I4.-Fig. 2 , b. (1) Orange ; (2) bright blue; (3) violet. The right edge hazy.

4. I6.- The left side blue, the right violet, with a narrow pink band on the lower edge.

4. I7. - The greater part of the cloud a very bright light blue, violet on the right, pink on the upper edge towards the left.

4.22. - Very faint and bluish.

4.25. - The cloud smaller, and bluish-green, but still faint.

4.26.-Green, and brighter.

4.29.-Fig. 2, c. The whole cloud much brighter, though not so bright as when first seen. (I) Reddish; (2) green; (3) reddish.

4.34. - The cloud the same shape as at 4.29 , and the whole of if orange-coloured. After this moment it was hidden by heavy clouds.

Beside the two clouds above described, and several other smaller ones similar to them, there was visible in the west, at 4.26 , a long narrow band of cloud (about $20^{\circ}$ long and $3^{\circ}$ or $4^{\circ}$ broad), parallel to the horizon, and of a distinctly violet colour throughout.

Sunderland, January i2

\section{Parallel Roads in Norway}

IN Mr. Hansen's account of the terrace formation of Central Norway he discards the sea theory of their origin, as well as the detrital dam, the local glacier theory, and also that of Prof. Prestwich, of landslips. The cause he ascribes to rests in the passage of the inland ice seawards, allowing lakes to form in the watershed while ice remained in the valleys seaward. Does this idea not reverse the order of Nature? Would it not be far simpler, more reasonable, and more in accordance with the laws of Nature to conclude that ice would remain in the highest valleys of the country longest, and that the parallel roads or terraces are the ice margins or lateral moraines where the ice rested after the most intense glaciation ceased, while the surplus passed over the cols, and the passage seaward was more or less retarded by the configuration of the country? The Lochaber roads are mostly composed of the usual glacial stuff of the district, it is neither washed as lake margins or sea beaches. The only water-washed material seems to have run down from the hills above, before glaciation ceased, and vegetation covered the surface. The roads are neither strictly parallel or horizontal, and just what might be expected to be formed by ice lying for a long time in a valley when the growth did not greatly exceed the waste and the motion was slow.

Edinburgh

JAMES MELVIN

\section{Dew}

HAVING read with interest the abstract in NATURE of January 14 (p. 256) of Mr. Aitken's ohservations on dew, I noted attentively during a walk this morning the behaviour of the hoar-frost as deposited on different objects. The morning was fine and frosty after a clear cold night. There was a copious deposit of hoar-frost upon the grass, upon the upper side of wooden rails, and upon the topmost twigs of the bushes in the tall hedges ( 6 to 8 feet high), but the lower twigs in the hedges had little or none. On stones in the road, as Mr. Aitken observes, there was little hoar-frost on the upper surface, only lines of ice crystals along the salient angles, but their under surfaces were thickly covered. With the loose heaps of brolien stones by the road-sides the case was different : here the uppermost stones were thickly coated with frost on their upper surface, but had little on their lower surface ; the stones underneath the uppermost layer, on the contrary, were coated with hoarfrost on their under, but not on their upper, surfaces. The hollow "cat's ice" on the road-side puddles, where previously 